Submitted to the Astronomical Journal

\title{
First Constraints on Rings in the Pluto System
}

\author{
A. J. Steffl and S. A. Stern \\ steffl@boulder.swri.edu \\ Southwest Research Institute, Space Science and Engineering Division \\ 1050 Walnut Street, Suite 400, Boulder, CO 80302
}

\begin{abstract}
Simple theoretical calculations have suggested that small body impacts onto Pluto's newly discovered small satellites, Nix and Hydra, are capable of generating time-variable rings or dust sheets in the Pluto system. Using HST/ACS data obtained on 2006 February 15 and 2006 March 2, we find no observational evidence for such a ring system and present the first constraints on the present-day $\mathrm{I} / \mathrm{F}$ and optical depth of a putative ring system. At the $1500-\mathrm{km}$ radial resolution of our search, we place a $3 \sigma$ upper limit on the azimuthally-averaged normal I/F of ring particles of $5.1 \times 10^{-7}$ at a distance of $42,000 \mathrm{~km}$ from the Pluto-Charon barycenter, the minimum distance for a dynamically stable ring (Stern et al. 1994; Nagy et al. 2006); $4.4 \times 10^{-7}$ at the orbit of Nix; and $2.5 \times 10^{-7}$ at the orbit of Hydra. For an assumed ring particle albedo of 0.04 (0.38), these I/F limits translate into $3 \sigma$ upper limits on the normal optical depth of macroscopic ring particles of $1.3 \times 10^{-5}\left(1.4 \times 10^{-6}\right), 1.1 \times 10^{-5}\left(1.2 \times 10^{-6}\right), 6.4 \times 10^{-6}\left(6.7 \times 10^{-7}\right)$, respectively. Were the New Horizons spacecraft to fly through a ring system with optical depth of $1.3 \times 10^{-5}$, it would collide with a significant number of potentially damaging ring particles. We therefore recommend that unless tighter constraints can be obtained, New Horizons cross the putative ring plane within 42,000 km of the Pluto-Charon barycenter, where rings are dynamically unstable. We derive a crude estimate of the lifetime of putative ring paritcles of 900 years.
\end{abstract}

Subject headings: planets and satellites: individual ( Pluto) — planets: rings Kuiper belt 


\section{Introduction}

The discovery of Pluto's two small ( $\sim 100 \mathrm{~km}$ diameter) satellites, Nix and Hydra, in 2005 (Weaver et al. 2006) raised the possibility that Pluto may possess a time-variable ring system (Stern et al. 2006a). Previously, Durda \& Stern (2000) demonstrated that collisions between small Kuiper belt debris and larger objects, such as Pluto's satellites, are a common occurrence. The characteristic ejecta velocity resulting from these collisions will be of order $1-10 \%$ of the impactor velocity, or $10-100 \mathrm{~m} \mathrm{~s}^{-1}$. Since the escape velocities of Nix and Hydra are between 30 and $90 \mathrm{~m} \mathrm{~s}^{-1}$, depending on their precise size and density, ejecta from such collisions can escape from Pluto's small satellites but will generally remain gravitationally bound to Pluto, thus forming rings. This is in contrast to the situation at Charon, where most such ejecta will fall back onto the surface, due to Charon's $\sim 500 \mathrm{~m} \mathrm{~s}^{-1}$ escape velocity. Such rings will be highly time-variable, as collisions on Nix and Hydra compete with loss processes. Stern et al. (2006a) calculated a characteristic ring optical depth of $\tau=5 \times 10^{-6}$ for ring material between Nix and Hydra. This is considerably more tenuous than the main rings of Saturn, Uranus, and Neptune, but comparable to the optical depth of Jupiter's rings (Burns et al. 1984) and the faint dust rings of Uranus (Showalter \& Lissauer 2006).

No prior observational constraints on the reflectance or optical depth of rings in the Pluto system have been published. We therefore undertook a search for such material using existing datasets acquired by the HST Advanced Camera for Surveys (ACS) in early 2006. In addition to the discovery potential of this search, detection of or constraints on Plutonian rings can yield useful information about both the potential hazards of ring material to the NASA New Horizons Pluto mission, now en route, and the small body population orbiting in the 30-50 AU region of the Kuiper belt traversed by Pluto.

In what follows we describe the dataset we used to constrain the amount of ring material in the Pluto system, the analysis techniques we employed, and the results we obtained. We then go on to interpret our results, which are upper limits, as they apply to ring system hazards for New Horizons and the lifetime of ring particles.

\section{Observations}

Immediately following the discovery of Nix and Hydra, our team obtained Director's Discretionary time to use HST to confirm the existence of the new satellites. However, to conserve the lifetime of the remaining functional HST gyros, HST began operations in 2-gyro mode before the confirmation observations could be executed. In this mode, most targets can only be observed when they are on the trailing side of the Sun as it moves along 
the ecliptic, thus precluding observations of the Pluto system until February 2006. When Pluto again became observable, it was targeted with two HST visits of one orbit each using the ACS High Resolution Channel (HRC) through Guest Observer/Director's Discretionary (GO/DD) program 10774. Selected observational parameters from these visits are presented in Table 1.

The first visit occurred on 2006 Feb 15.7 UT. During this visit, four long exposures (475second integrations) with the F606W filter (broad V) were obtained using a non-integer dither box pattern to minimize the effect of bad detector pixels and improve the spatial sampling. In these long exposures, both Pluto and Charon are saturated with a small amount of bleeding along the CCD columns. To aid the registration of the long exposures, short, unsaturated exposures (1-second integrations) were also obtained at each point in the dither pattern. Both Nix and Hydra were clearly detected at high signal to noise (Mutchler et al. 2006), and no additional satellites were detected between the orbits of Nix and Hydra down to a 90\%-confidence limiting magnitude of $\mathrm{V}=25.7$ (Steffl et al. 2006).

With the successful confirmation of both satellites from analysis of archival ACS data (Buie et al. 2006) and the 2006 February 15 visit, the observational setup of the second visit, which occurred on 2006 March 2, was modified to also obtain the first $B-V$ colors of Nix and Hydra (Stern et al. 2006b). A three-point, non-integer dither pattern was employed with a $145 \mathrm{~s}$ exposure using the F606W filter and a $475 \mathrm{~s}$ exposure using the F435W (Johnson B) obtained at each point of the dither pattern. A $3 \mathrm{~s}$ exposure using the F435W filter and a $1 \mathrm{~s}$ exposure using the $\mathrm{F} 606 \mathrm{~W}$ filter were also obtained at points 1 and 3 of the dither pattern, respectively, to aid in image registration. Since the point spread function (PSF) of the ACS varies significantly between filters, data taken during the 2006 March 2 visit with the F606W filter were analyzed separately from data taken with the F435W filter.

For each visit, the exposures made using a given filter were "drizzled" together using the PyRAF Multidrizzle procedure (Koekemoer et al. 2002). Via this procedure, the individual images are corrected for the geometric distortion of the ACS instrument, rotated so that north is up and east to the left, sky background subtracted, co-registered relative to Pluto using the non-saturated 1-s exposures, and combined using a median filter. Detector pixels that have anomalously low sensitivity, high dark counts, or are saturated were flagged and excluded from further analysis. The median combination removes artifacts, such as cosmic ray events or star trails, that do not appear in same position relative to Pluto in at least two of the images. The resulting images from the February and March visits using the F606W filter are shown in Figure 1.

Pluto is highly saturated in the long integrations obtained during both visits. Charon is also saturated in the long integrations obtained during the February visit. As a result, the 
two-dimensional pattern of flux from Pluto and Charon dominates over the sky background, even several arcseconds away from Pluto. Complex structure can be seen in this pattern, as a result of the higher-order behavior in the wings of the ACS HRC point spread function $(\mathrm{PSF})$.

\section{Results}

To reduce the strong background gradients in the processed images due to Pluto and Charon, we employ the high-pass filter method of Showalter \& Lissauer (2006). At each pixel in the image, a first-order, two-dimensional polynomial (i.e. a tilted plane) is fit to the pixels lying within a surrounding circular region. A small circular aperture at the center of this region is excluded from the fit so as not to reduce the intensity of point features. A radius of 12 pixels was chosen for the outer limit of the fit; a radius of 5 pixels was chosen for the inner aperture, to enclose $80 \%$ of the flux from a point source (Sirianni et al. 2005). The value of the fit is then subtracted from the central pixel and the process repeated for all pixels in the drizzled images. The resulting filtered images still contain the high-frequency components of the 2-D pattern of flux from Pluto and Charon, but the low-frequency components are much reduced. Visual inspection of the filtered images reveals no immediate evidence for a faint ring system. As noted by Showalter \& Lissauer (2006), this high-pass filter method is not photometrically accurate (tending to narrow bright features), so an alternative method must be used to place quantitative limits on the I/F of a potential ring system.

Flux from Pluto and Charon is the dominant source of counts in the region of the images where a ring system might exist. To zeroth order, the PSFs of the ACS HRC are azimuthally symmetric. However, the long integration times used in these observations allow the non-symmetric components of the PSF, i.e., the wings, to rise significantly above the level of the sky background. The situation is also complicated by the fact that neither Pluto nor Charon are true point sources, having apparent angular diameters of 0 '!104 and and 0'052, respectively, during the February visit (for comparison, the pixel size of the ACS HRC is approximately $\left.0^{\prime \prime} 025\right)$. Pluto is also known to exhibit significant albedo variations across its surface (Stern et al. 1997; Young et al. 2001). As a result, the observed pattern of flux from Pluto and Charon can not be accurately removed by the simple subtraction of an azimuthally-averaged image from the data. In principle, more sophisticated techniques, e.g. PSF subtraction, can be used to remove this pattern; however, outside of the PSF core, the observed non-symmetric, high-frequency variations in flux from Pluto and Charon are poorly described by model ACS PSFs, such as those generated by the Tiny Tim program (Krist \& Hook 2004). Since neither the 2-D pattern of flux from Pluto and Charon nor the 
spatial extent of a putative ring system are known a priori, attempts to characterize and remove Pluto and Charon's flux using fits to the data run a significant risk of inadvertently removing some or all of the signal from a potential ring system. Thus, given the difficulty of removing the background flux from Pluto and Charon without introducing artifacts to the data, we have adopted a simple approach: assuming that all of the flux contained in the image is due to light backscattered from a ring system. This is clearly an oversimplification, but it yields a firm upper limit on the reflectance of a putative ring system.

Since the orbits of Pluto's three known moons are all essentially co-planar (Weaver et al. 2006; Buie et al. 2006), any ring system would be expected to share this plane. We therefore divided the plane of Charon's orbit into 100 circular annuli centered on the Pluto-Charon barycenter. We chose a width of $1500 \mathrm{~km}$ for the annuli, based on the projected size of three of the 0 .'025 HRC pixels at Pluto. Our results, however, are only weakly dependent on the choice of annulus width. Areas of the image containing diffraction spikes from either Pluto or Charon were masked out and excluded from further analysis, as were circular regions surrounding Pluto, Charon, Nix, and Hydra. Pluto's diffraction spikes provide a natural means of dividing the image into quadrants. Quadrant boundaries were used to divide each annulus into four segments. The total count rate contained in and solid angle subtended by each annulus segment within a given quadrant was then calculated.

The standard measurement of reflectance for planetary rings is the dimensionless ratio $\mathrm{I} / \mathrm{F}$, where $\mathrm{I}$ is the observed intensity and $\pi \mathrm{F}$ is the incident (solar) flux. For an optically thin ring, $\mathrm{I} / \mathrm{F}$ is proportional to $1 / \sin (\mathrm{B})$, where $\mathrm{B}$ is the ring opening angle, defined as the angle between the line of sight and the ring plane. We therefore multiply $\mathrm{I} / \mathrm{F}$ by $\sin (\mathrm{B})$ to remove this geomterical factor, yielding the "normal I/F", i.e., that seen from an observer looking perpendicular to the ring plane at the same phase angle. The normal I/F is given by the following equation:

$$
\left(\frac{I}{F}\right)_{\perp}=\frac{C r^{2} \sin (B)}{\pi \Omega} 10^{\left(m_{\odot}+\Delta m(\alpha)-z\right) / 2.5}
$$

where $C$ is the total count rate observed in the segment(s) of an annulus lying within a particular quadrant, in electrons $\mathrm{s}^{-1} ; r$ is the distance from the Sun to Pluto in AU; $\Omega$ is the solid angle subtended by the annulus segment(s); $B$ is the ring opening angle, defined as the angle between the line of sight and the ring plane; $m_{\odot}=-26.70$ is the apparent magnitude of the Sun at a distance of 1 AU using the HRC F606W filter bandpass (Colina et al. 1996); $\Delta m(\alpha)$ is the difference in magnitude between opposition and phase angle $\alpha$; and $z=26.13$ is the magnitude system zero point (Sirianni et al. 2005). Since the phase function of the putative ring system is unknown, we have adopted the phase function used by Karkoschka 
(2001) to model the rings of Uranus:

$$
\Delta m(\alpha)=\beta \alpha+0.5 \alpha /\left(\alpha_{0}+\alpha\right)
$$

The first term of this equation is the standard linear dependence with a phase coefficient of $\beta=0.03 \mathrm{mag} \mathrm{deg}^{-1}$. The second term represents the opposition surge with a width at half maximum of $\alpha_{0}=8 \mathrm{deg}^{-1}$. For the phase angle of $\alpha=1.69^{\circ}$ during the February visit, these two terms result in a correction of 0.14 mag.

If Pluto's rings are more tightly confined radially than the $\sim 1500 \mathrm{~km}$ resolution of our search, Eq. 1 will underestimate the true I/F. However, given the apparent lack of any additional small satellites in the Pluto system (Steffl et al. 2006), it is difficult to see how a ring system could remain so tightly confined radially.

Profiles of the normal I/F versus distance from the Pluto-Charon barycenter, as derived from the two HST visits, are shown in Figure 2. In all quadrants, the derived I/F value falls off sharply with increasing distance from Pluto, evidence that light from Pluto dominates the total flux contained in the annulus segments. There is no statistically significant evidence for a ring system in any of the I/F profiles. Profiles from the 2006 March 2 visit using the $\mathrm{F} 435 \mathrm{~W}$ filter have a significantly higher I/F value at a given barycentric distance (owing to the broader PSF with this filter), and therefore were not used in further analysis.

The inclination of the line of sight to the orbital plane of Pluto's moons causes the circular annuli to appear elliptical when projected onto the plane of the sky. As a result, annulus segments appear closer to Pluto in quadrants II and IV than in quadrants I and III. Thus, I/F upper limits derived from these quadrants are significantly higher, as is readily seen in Figure 1. During the 2006 February 15 visit, quadrant III provides the tightest constraint on the normal I/F of a putative ring system between the orbits of Nix and Hydra, while during the 2006 March 2 visit, quadrant I provides the tightest constraint. However, the statistical error associated with the I/F profile from quadrant III of the February visit is significantly lower than that from quadrant I of the F606W data from the March visit, owing to the factor of 4 greater integration time and better rejection of star trails and cosmic rays events during the February visit.

Dynamical interactions with Charon render orbits about the Pluto-Charon barycenter with semi-major axes less than 42,000 km (2.15 times the orbital semi-major axis of Charon) unstable (Stern et al. 1994; Nagy et al. 2006). The $3 \sigma$ upper limits on the normal I/F of a putative ring are given at this inner boundary and the orbits of Nix and Hydra in Table 2.

Assuming that macroscopic particles dominate the backscatter from the ring, the normal 
optical depth of the ring can be derived via the following equation:

$$
(I / F)_{\perp}=p \tau_{\perp}
$$

where $p$ is the geometric albedo of the particles. To provide an upper limit on the normal optical depth of macroscopic ring particles, we assume a very dark ring particle albedo of $p_{V}=0.04$, similar to particles in the rings of Uranus (Karkoschka 2001). We also calculate normal optical depth limits for an assumed macroscopic ring particle albedo as high as Charon's, i.e., $p_{V}=0.38$ (Buie et al. 1997). The derived upper limits on normal optical depth are shown versus barycentric distance in Figure 3, and the values of these upper limits at the minimum stable barycentric distance, Nix's orbital distance, and Hydra's orbital distance are presented in Table 2.

\section{Implications}

Based on the HST observations of Pluto in February and March 2006, the Pluto system does not currently have rings with a normal $\mathrm{I} / \mathrm{F}$ in excess of $5.1 \times 10^{-7}$ or a normal optical depth greater than $1.3 \times 10^{-5}$. This implies that the current Plutonian ring system, if it exists, is either tenuous, like Jupiter's ring system, or is tightly confined to less than $1500 \mathrm{~km}$ in width, below the spatial resolution of our search. However, given that there are no additional satellites of the Pluto system with an effective diameter larger than $16 \mathrm{~km}$ (Steffl et al. 2006), it is difficult to imagine how such a narrow ring could be formed or maintained. While usefully constraining, the derived limits on the normal optical depth of macroscopic ring particles are still somewhat higher than the typical, time-averaged optical depth of $\tau=5 \times 10^{-6}$ predicted by Stern et al. (2006a).

We next comment on what our results imply regarding hazards for the New Horizons spacecraft traversing the Pluto system. Assuming a unimodal size distribution of ring particles, the number of particles that would impact the spacecraft is given by:

$$
N_{p}=\tau \sec \theta\left(\sigma_{N H} / \sigma_{p}\right)
$$

where $\theta$ is the inclination of the spacecraft trajectory with the ring plane, $\sigma_{N H}$ is the crosssectional area of the spacecraft, and $\sigma_{p}$ is the cross-sectional area of a ring particle. For a ring optical depth of $\tau=1.3 \times 10^{-5}$, a trajectory inclined $30^{\circ}$ to the ring plane, a spacecraft projected cross sectional area of $\sigma_{N H} \approx 10 \mathrm{~m}^{2}$, and a unimodal distribution of $1 \mu \mathrm{m}$ diameter ring particles, we find $2 \times 10^{8}$ impacts on the spacecraft, with a total impactor mass of $1 \times 10^{-4} \mathrm{~g}$, 
assuming a ring particle density, $\rho_{p}$, of $1 \mathrm{~g} \mathrm{~cm}^{-3}$. New Horizons is robust enough to withstand collisions with $1 \mu \mathrm{m}$ particles at its Pluto flyby speed of $\sim 12 \mathrm{~km} \mathrm{~s}^{-1}$. However, collisions with $100 \mu \mathrm{m}$ diameter particles are potentially damaging; for a unimodal ring of $100 \mu \mathrm{m}$ particles, up to $2 \times 10^{4}$ such collisions could be expected.

Our ring constraints are not tight enough (by several orders of magnitude) to ensure the safety of the New Horizons spacecraft, were it to cross the ring plane at a distance where ring particles might be present. In the absence of tighter constraints on the optical depth of a putative ring system, we therefore recommend that New Horizons cross the ring plane within $42,000 \mathrm{~km}$ of the system barycenter, where dynamical interactions with Charon prevent stable orbits (Stern et al. 1994; Nagy et al. 2006). The current planned trajectory for New Horizons is safely within this distance.

Finally, we can use our ring optical depth constraint to estimate the lifetime of ring particles at Pluto. First, we point out that the re-collision timescale for ring particles orbiting between Nix and Hydra, i.e., their timescale to be reabsorbed by their parent satellite can be computed following Burns et al. (1984), yielding a timescale of order a few $10^{3}$ years. Effectively, this is an upper limit for ring particle lifetimes since other loss processes would only speed up the loss timescale.

Making the assumption of a steady state ring population, where particle production and loss are in balance over long time scales, we can derive a separate ring particle lifetime, $T_{p}$, based on our derived ring particle optical depth:

$$
T_{p}=M_{R} /\left(d M_{R} / d t\right)
$$

where $M_{R}$ is the ring mass, which can be computed from the optical depth constraint $\tau$ as:

$$
M_{R}=(8 / 3) \pi r_{p} \rho_{p} \tau R d R
$$

where $r_{p}$ is the characteristic ring particle radius, and $R$ and $d R$ are the ring's radius and width. $d M_{R} / d t$ can be written very simply as:

$$
d M_{R} / d t=2 \gamma M_{s a t} / T_{S S}
$$

where $M_{\text {sat }}$ is the average mass of Nix and Hydra, $T_{S S}$ is the age of the solar system, and $\gamma$ is the fractional mass of the satellites that have been lost to erosion. Thus, we find: 


$$
T_{p}=r_{p} \rho_{p} \tau T_{S S} R d R / \gamma r_{\text {sat }}^{3} \rho_{\text {sat }}
$$

For a characteristic radius of $50 \mathrm{~km}$ and density of $2 \mathrm{~g} \mathrm{~cm}^{-3}$ for Nix and Hydra, $\gamma=10^{-4}$ (consistent with Durda \& Stern [2000]), and a unimodal ring of $1 \mu \mathrm{m}$ diameter particles with density $1 \mathrm{~g} \mathrm{~cm}^{-3}$ extending from Nix's orbit to Hydra's orbit, i.e., from 48,675-64,780 km (Buie et al. 2006), we find a crude ring particle lifetime constraint of $T_{p}=900 \mathrm{yr}$.

\section{Conclusions}

We have used existing HST ACS observations of the Pluto system to derive the first constraints on the normal I/F and optical depth of rings having a radial extent greater than $1500 \mathrm{~km}$. We find a $3 \sigma$ upper limit of $I / F_{\perp}=5.1 \times 10^{-7}$ and, assuming a macroscopic ring particle albedo of $0.04(0.38)$, a $3 \sigma$ upper limit $\tau_{\perp}=1.3 \times 10^{-5}\left(1.4 \times 10^{-6}\right)$. Although higher ring optical depths are possible for tightly confined rings less than $1500 \mathrm{~km}$ wide, this is unlikely given the apparent lack of additional small satellites to shepherd the rings. The present-day optical depth limits on the rings of Pluto are usefully constraining, but they remain a factor of three larger than the characteristic ring optical depth of $5 \times 10^{-6}$ predicted by Stern et al. (2006a). We note that the optical depth constraint derived from the HST observations is not sufficient to ensure safe passage of the New Horizons spacecraft through the ring plane at distances of 42,000-65,000 km. Finally, in the case of a ring in steady state, we estimate a ring particle lifetime of 900 years.

Financial support for this work was provided by NASA through grant numbers GO-10427 and GO-10774 from the Space Telescope Science Institute, which is operated by the Association of Universities for Research in Astronomy, Inc., under NASA contract NAS5-26555. Additional support was provided by the New Horizons Pluto-Kuiper Belt mission. We thank M. Bullock, L. Young and an anonymous referee for reading and commenting on this manuscript.

\section{REFERENCES}

Buie, M. W., Tholen, D. J., \& Wasserman, L. H. 1997, Icarus, 125, 233

Buie, M. W., Grundy, W. M., Young, E. F., Young, L. A., \& Stern, S. A. 2006, AJ, 132, 290

Burns, J. A., Showalter, M. R., \& Morfill, G. E. 1984, in IAU Colloq. 75: Planetary Rings, ed. R. Greenberg \& A. Brahic, 200-272 
Colina, L., Bohlin, R. C., \& Castelli, F. 1996, AJ, 112, 307

Durda, D. D. \& Stern, S. A. 2000, Icarus, 145, 220

Karkoschka, E. 2001, Icarus, 151, 51

Koekemoer, A. M., Fruchter, A. S., Hook, R., \& Hack, W. 2002, in HST Calibration Workshop, 337

Krist, J. \& Hook, R. 2004, The Tiny Tim User's Guide version 6.3, http://www.stsci.edu/software/tinytim/tinytim.pdf

Mutchler, M. J., Steffl, A. J., Weaver, H. A., Stern, S. A., Buie, M. W., Merline, W. J., Spencer, J. R., Young, E. F., \& Young, L. A. 2006, IAU Circ., 8676, 1

Nagy, I., Süli, Á., \& Érdi, B. 2006, MNRAS, 370, L19

Showalter, M. R. \& Lissauer, J. J. 2006, Science, 311, 973

Sirianni, M., Jee, M. J., Benítez, N., Blakeslee, J. P., Martel, A. R., Meurer, G., Clampin, M., De Marchi, G., Ford, H. C., Gilliland, R., Hartig, G. F., Illingworth, G. D., Mack, J., \& McCann, W. J. 2005, PASP, 117, 1049

Steffl, A. J., Mutchler, M. J., Weaver, H. A., Stern, S. A., Durda, D. D., Terrell, D., Merline, W. J., Young, L. A., Young, E. F., Buie, M. W., \& Spencer, J. R. 2006, AJ, 132, 614

Stern, S. A., Parker, J. W., Duncan, M. J., Snowdall, J. C. J., \& Levison, H. F. 1994, Icarus, 108,234

Stern, S. A., Buie, M. W., \& Trafton, L. M. 1997, AJ, 113, 827

Stern, S. A., Weaver, H. A., Steffl, A. J., Mutchler, M. J., Merline, W. J., Buie, M. W., Young, E. F., Young, L. A., \& Spencer, J. R. 2006a, Nature, 439, 946

Stern, S. A., Mutchler, M. J., Weaver, H. A., \& Steffl, A. J. 2006b, ArXiv Astrophysics e-prints

Weaver, H. A., Stern, S. A., Mutchler, M. J., Steffl, A. J., Buie, M. W., Merline, W. J., Spencer, J. R., Young, E. F., \& Young, L. A. 2006, Nature, 439, 943

Young, E. F., Binzel, R. P., \& Crane, K. 2001, AJ, 121, 552 
Table 1. Selected Observational Parameters

\begin{tabular}{lcccc}
\hline \hline Observation Date $(\mathrm{UT})^{\mathrm{a}}$ & $\mathrm{r}(\mathrm{AU})$ & $\Delta(\mathrm{AU})$ & $\alpha(\mathrm{deg})$ & $\mathrm{B}(\mathrm{deg})$ \\
\hline 2006 Feb 15.659 & 31.07 & 31.54 & 1.59 & 37.2 \\
2006 Mar 02.747 & 31.08 & 31.31 & 1.77 & 37.5 \\
\hline
\end{tabular}

${ }^{a}$ Midpoint of observation

Note. $-r$ is Pluto's heliocentric distance, $\Delta$ is Pluto's geocentric distance, $\alpha$ is Pluto's phase angle (Sun-Pluto-Earth angle), and $B$ is the ring plane opening angle.

Table 2. Selected $3 \sigma$ Upper Limits on the Putative Rings

\begin{tabular}{lccc}
\hline \hline Barycentric Distance $(\mathrm{km})$ & $(\mathrm{I} / \mathrm{F})_{\perp}$ & $\tau_{\perp}, \mathrm{p}=0.04$ & $\tau_{\perp}, \mathrm{p}=0.38$ \\
\hline 42,000 (Min. stable dist.) & $5.1 \times 10^{-7}$ & $1.3 \times 10^{-5}$ & $1.4 \times 10^{-6}$ \\
48,675 (Nix) & $4.4 \times 10^{-7}$ & $1.1 \times 10^{-5}$ & $1.2 \times 10^{-6}$ \\
64,780 (Hydra) & $2.5 \times 10^{-7}$ & $6.4 \times 10^{-6}$ & $6.7 \times 10^{-7}$ \\
\hline
\end{tabular}




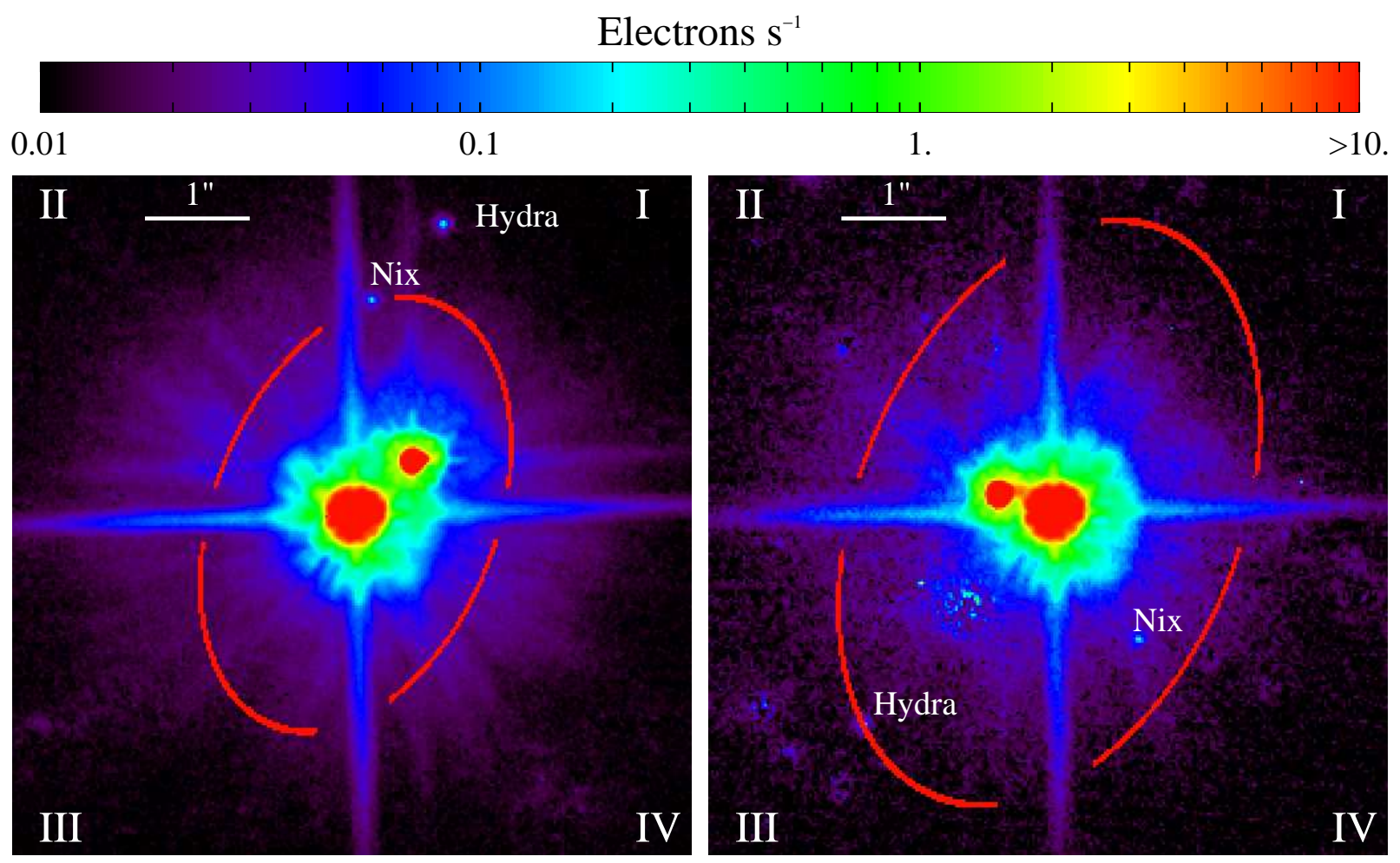

Fig. 1.- Drizzled HRC F606W images of the Pluto system. The image on the left is from the 2006 February 15 visit, while the image on the right is from the 2006 March 2 visit. Pluto, Charon, Nix, and Hydra can be clearly seen. All other features are due to the extended PSF halos around Pluto and Charon or artifacts introduced by the incomplete removal of background star trails or cosmic ray events. The diffraction spikes from Pluto have been used to divide each image into four quadrants. $1500 \mathrm{~km}$ width annuli at the orbital distances of Nix (left) and Hydra (right) are shown in red. Four $10^{\circ}$ segments (half width), centered on each diffraction spike, are excluded from analysis. A one arcsecond scale bar is shown in the upper left of each image. 


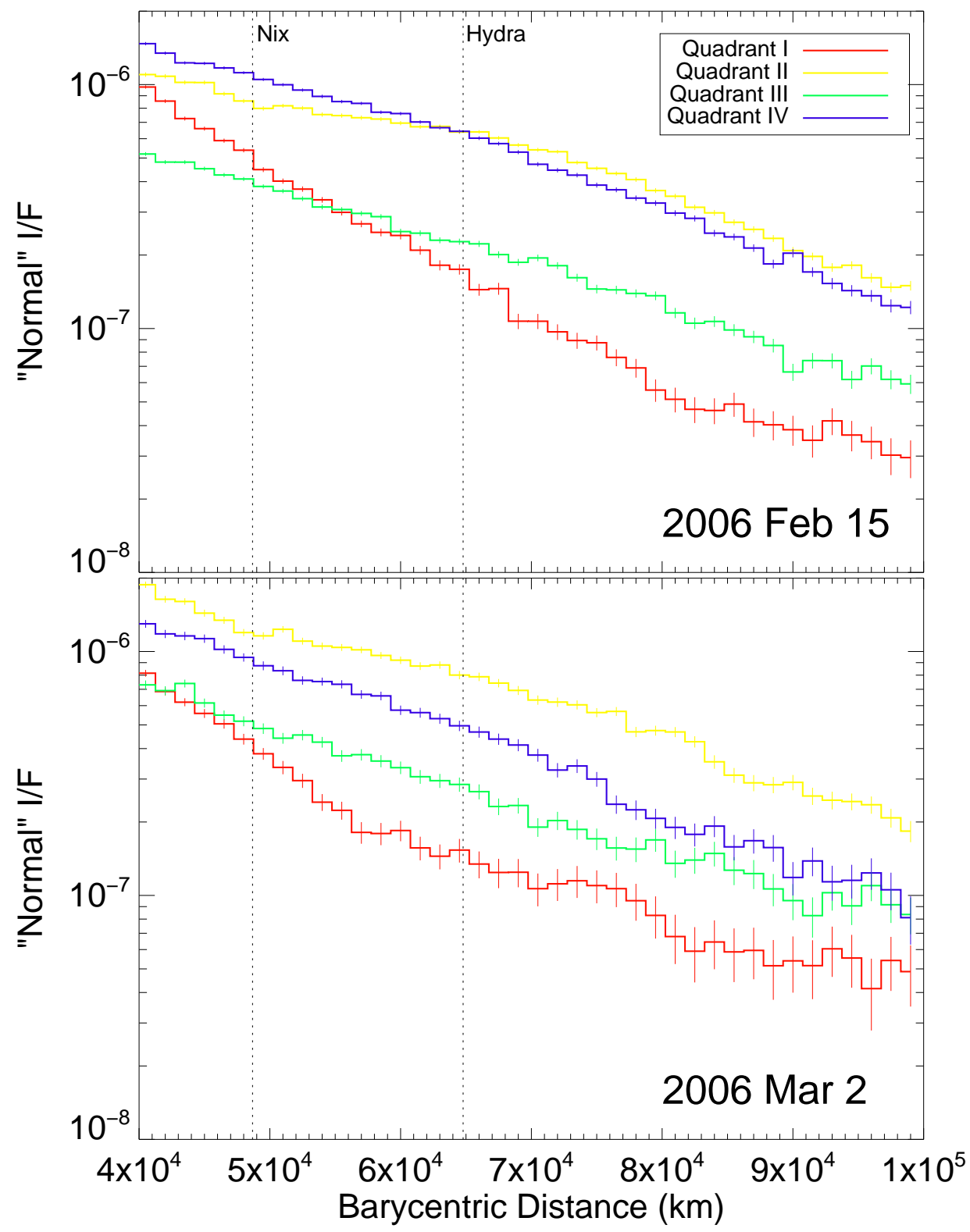

Fig. 2.- "Normal" I/F as a function of barycentric distance, assuming all observed flux is backscattered from a ring system. $1 \sigma$ error bars are shown at each point. For a given barycentric distance, annulus segments in quadrants I and III appear at a greater angular distance from Pluto, owing to the projection of the putative ring plane on the plane of the sky. Since the observed flux in a given annulus segment is dominated by flux from Pluto, these quadrants provide the tightest constraints on the I/F of a putative ring system. Normal I/F values shown for the 2006 March 2 visit are derived exclusively from data using the F606W filter, as the normal I/F values derived from the F435W data are significantly higher. The orbital semi-major axes of Nix and Hydra are shown by vertical dotted lines at 48,675 and $64780 \mathrm{~km}$, respectively. 


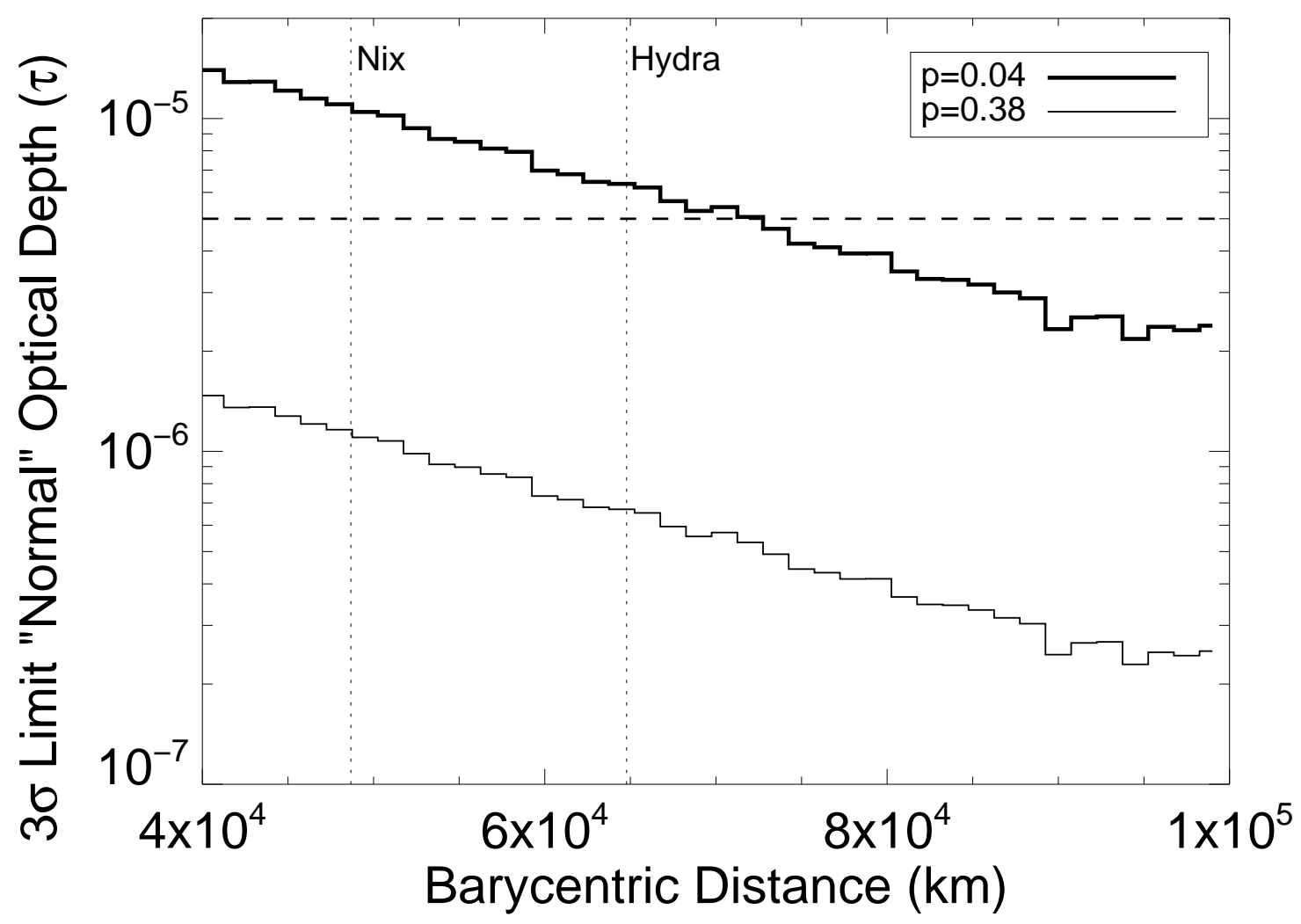

Fig. 3.- $3 \sigma$ upper limit on "normal" optical depth for a as a function of barycentric distance for assumed macroscopic ring particle albedos of 0.04 (thick line) and 0.38 (thin line). The optical depth limit is derived from the normal I/F limit from quadrant III of the 2006 February 15 visit. The horizontal dashed line marks the characteristic ring optical depth predicted by Stern et al. (2006a). Orbital semi-major axes of Nix and Hydra are shown by vertical dotted lines at 48,675 and $64,780 \mathrm{~km}$, respectively. 